\title{
Relativistic aspects of Earth's rotation
}

\author{
Michael Soffel and Sergei A. Klioner \\ Lohrmann Observatory, Dresden Technical University, D-01062 Dresden, Germany \\ email: soffel,klioner@rcs.urz.tu-dresden.de
}

\begin{abstract}
We discuss relativistic aspects of Earth's roration
Keywords. astrometry, reference systems, relativity, Earth
\end{abstract}

Various relativistic aspects related with Earth's rotation are reviewed (see also Soffel \& Klioner 2005).

First the problem of reference systems is discussed, where the BCRS (barycentric celestial reference system) and GCRS (geocentric celestial reference system) act as basic systems. For the near future the first post-Newtonian approximation to Einstein's theory of gravity will be sufficient.

This does not only apply to the problem of reference systems but also to the construction of global quantities such as angular velocity, moments of inertia etc., and to the corresponding dynamical equations of motion. In a series of papers, Damour, Soffel \& $\mathrm{Xu}(1991,1992,1993)$ have constructed an improved post-Newtonian celestial mechanics with the BCRS and GCRS as basis. The dynamical equation for rotational motion of the Earth is given explicitly with full post-Newtonian expression for the torque in the GCRS. For practical applications a model of rigidly rotating multipoles has been suggested (Klioner et al. 2001). This approach is presently pursued to derive an improved consistent post-Newtonian nutation series.

In a series of papers $\mathrm{Xu}$ and coworkers laid the foundation for a relativistic description of elastic deformable rotating astronomical bodies (Xu et al. 2001, 2003, 2005). This local approach still poses fundamental problems since the relation of the formalism with observed quantities related with Earth's rotation is still unclear.

\section{References}

Damour, T., Soffel, M., \& Xu, C. 1991, Phys. Rev., D 43, 3273

Damour, T., Soffel, M., \& Xu, C. 1992, Phys. Rev., D 45, 1017

Damour, T., Soffel, M., \& Xu, C. 1993, Phys. Rev., D 47, 3124

Klioner, S., et al. 2001, in: N. Capitaine (ed.), Proc. Journées 2001 (Paris: Observatoire)

Soffel, M., \& Klioner, S. 2005, in: N. Capitaine (ed.), Proc. Journées 2004 (Paris: Observatoire)

Xu, C., Wu, X., \& Soffel, M. 2001, Phys. Rev., D 63, 043002

Xu, C., Wu, X., Soffel, M., \& Klioner, S. A. 2003, Phys. Rev., D 68, 064009

Xu,C., Wu, X., \& Soffel, M. 2005, Phys. Rev., D 71, 024030 